\title{
Causas de las desigualdades territoriales en la fecundidad: un estudio a escala metropolitana en el área barcelonesa ${ }^{1}$
}

\author{
Jordi Bayona-i-Carrasco², Miguel Rubiales Pérez ${ }^{3}$, Fernando Gil-Alonso ${ }^{4}$ \\ y Isabel Pujadas Rúbies ${ }^{5}$
}

\begin{abstract}
RESUMEN
Los comportamientos demográficos están experimentando una creciente segmentación en el interior de las regiones metropolitanas. Por ejemplo, los municipios de la Región Metropolitana de Barcelona (RMB) presentan diferentes intensidades de fecundidad, valores que incluso difieren en mayor grado que entre las provincias españolas. La principal hipótesis de este trabajo es que dichas diferencias se sustentan en la migración interna de parejas jóvenes ligadas al ciclo de vida, aunque la presencia de población extranjera o la renta media de los municipios también podrían influir. Para verificar estas hipótesis se ha calculado los índices sintéticos de fecundidad de 26 sub-áreas de la RMB, complementándose este análisis descriptivo con modelos de regresión lineal para los años 2001 y 2009. Los resultados confirman parcialmente la hipótesis principal, aunque en 2009 la complejidad residencial producto de la inmigración internacional añade la presencia de extranjeros (africanos especialmente) como factor explicativo de los altos niveles de fecundidad.
\end{abstract}

Palabras clave: Región metropolitana, fecundidad, suburbanización, inmigración extranjera, España.

\begin{abstract}
Demographic behaviour within metropolitan areas is increasingly spatially segmented, and fertility is a good example of this, as its growing spatial differences would depend on the type of metropolitan municipality analysed. The article's main hypothesis is that fertility level differences would mainly be explained by selective internal migration flows associated to young couples' life cycle. Alternative hypotheses are that fertility differences between municipalities could be explained by the share of foreign women living in them, o by disposable household income differences. In order to verify these hypotheses, the article analyses Barcelona Metropolitan Region's intra-metropolitan fertility. A preliminary descriptive analysis -total fertility rates (TFR) for 26 metropolitan subareas have been calculated-is completed by linear regression models for years 2001 and 2009. Results confirm that fertility differences are partly explained by young Spanish couples' internal migration flows towards small and medium suburban localities, but also by specific foreign groups, particularly Africans.
\end{abstract}

Key words: Metropolitan region, fertility, suburbanization, foreign migration, Spain.

Este trabajo es un resultado del Proyecto I+D Desigualdad social, polarización territorial y formación de espacios vulnerables en las grandes áreas metropolitanas españolas CSO2015-65219-C2-1-R (MINECO/FEDER, UE), financiado por el Ministerio español de Economía y Competitividad y los fondos FEDER de la Unión Europea, y dirigido por el Dr. Fernando Gil y la Dra. Isabel Pujadas, quien también coordina el grupo de investigación Territori, Població i Ciutadania, financiado por la Generalitat de Catalunya (GRC_2014SGR380) y del que forman parte los autores del artículo. Artículo recibido el 10 de octubre de 2015, aceptado el 10 de mayo de 2016 y corregido el 21 de junio de 2016. Centre d'Estudis Demogràfics / Departamento de Geografía, Universidad de Barcelona (España). E-mail: jordibayona@ub.edu Departamento de Geografía, Universidad de Barcelona (España).E-mail: mrubiales@ub.edu Departamento de Geografía, Universidad de Barcelona (España) E-mail: fgil@ub.edu

Departamento de Geografía, Universidad de Barcelona (España).E-mail: ipujadas@ub.edu 
A inicios del siglo XXI, la mayoría de las regiones metropolitanas se encuentran inmersas en un proceso de creciente fragmentación, siendo social y territorialmente cada vez menos homogéneas, con un aumento paulatino de la polarización social. Si bien los procesos de suburbanización, fragmentación y segregación, ya por parte de las clases más desfavorecidas como de las clases más altas, son un ejemplo de ello y se encuentran profundamente estudiados, con múltiples ejemplos en el caso europeo (Berger, 2004; Champion, 2001, 2002; Cheshire, 2006; Feria, 2011; Fielding, 1989; Indovina et al., 2007; Kabisch \& Haase, 2011, Leal, 2003; Monclús, 1998; Rérat, 2011) o latinoamericano (Aguilar, 2004; Aguilar y Mateos, 2011; Borsdorf, 2003; Dematteis, 1998; Sabatini y Brain, 2008, entre otros muchos autores), en cambio algunos de los aspectos que caracterizan esta división del espacio, como son los comportamientos demográficos, han sido objeto de menor atención, a pesar de que no dejan de ser un fiel reflejo de la división del espacio. Por ejemplo, ¿por qué razón, dentro de una misma región metropolitana, unos municipios tienen unos niveles de fecundidad más elevados que otros municipios vecinos? Precisamente el objetivo de este trabajo es analizar la evolución y las causas de la diferenciación espacial de la fecundidad en el interior de una área urbana española, tomando como caso de estudio la Región Metropolitana de Barcelona (RMB de ahora en adelante), que ha conocido durante los últimos años profundos procesos de cambio, ya sea por la continuación del proceso de suburbanización y desconcentración de la población, como por la llegada de un importante stock de inmigrantes extranjeros coincidiendo con el boom inmigratorio que experimentó el país. Particularmente, el caso de la RMB es ejemplar en cuanto dispone de un espacio central (el municipio de Barcelona) relativamente pequeño $\left(100 \mathrm{~km}^{2}\right)$ y bien delimitado, con lo cual los fenómenos demográficos relacionados con la centralidad se encuentran muy bien definidos. En este sentido, la principal hipótesis es que las diferencias intrametropolitanas de fecundidad se deben a la migración interna de parejas jóvenes -desde el núcleo central hacia los municipios periféricos- ligadas al ciclo de vida, aunque las diferencias de renta media entre los municipios o sus porcentajes de población extranjera -con desiguales pautas de fecundidad- también podrían ser variables explicativas.

El artículo desarrolla en primer lugar el marco analítico y el estado de la cuestión, incidiendo en aquellos estudios que abordan desde un punto de vista geográfico (y demográfico) las pautas de diferenciación de la fecundidad. Tras presentar las fuentes de datos estadísticos y la metodología utilizada, el texto pasa a describir las diferencias internas en la fecundidad de la RMB durante los 25 años transcurridos en el periodo 1986-2012. Posteriormente, y mediante un modelo de regresión lineal, se aportan elementos interpretativos a la creciente diferenciación de comportamientos, utilizando dos años (2001 y 2009) como puntos de análisis, y empleando como variables explicativas aquellas dinámicas metropolitanas que, como la migración interna y la llegada de población extranjera, han incidido con mayor peso en la Región Metropolitana de Barcelona durante los últimos años, a las que añadiremos las diferencias de renta familiar disponible de los municipios como otro posible factor de incidencia sobre la fecundidad. Las conclusiones y algunos aspectos de discusión que consideramos relevantes clausuran el artículo.

\section{Los procesos demográficos a escala metropolitana}

El crecimiento reciente de la fragmentación de los territorios metropolitanos viene asociado, en España, a dos fenómenos de distinta intensidad y recorrido temporal. Por un lado, el proceso de suburbanización, que se acentúa en los años setenta en las metrópolis más maduras (Barcelona y Madrid) y que paulatinamente se extiende a la mayoría de ciudades, tanto grandes como 
medianas, incluso pequeñas cabeceras comarcales que reproducen situaciones similares. Por el otro, la inmigración internacional, que aparece a finales de los años noventa con cierta intensidad en las zonas más dinámicas del país, provocando fuertes crecimientos metropolitanos, con centros que después de años de declive recuperan población y periferias que crecen a ritmos desenfrenados. Entre 2001 y 2011 en las 15 mayores áreas metropolitanas españolas la población crece en 3 millones de habitantes (desde los 19 millones iniciales), 2 de los cuales con nacionalidad extranjera (Gil-Alonso et al., 2016). En esas mismas áreas, las periferias ganan 2 millones de nuevos residentes (uno de los cuales de nacionalidad española), por uno los centros metropolitanos (que siguen perdiendo españoles). Ambos fenómenos, hasta el estallido de la crisis económica en 2008 y del mercado inmobiliario, caracterizan en mayor o menor intensidad las ciudades españolas. Como resultado, crece la diversidad, y se intensifican los procesos de transformación urbana.

El fenómeno de suburbanización residencial, que implica la salida de población desde los centros densos hacia sus periferias, viene determinado por el ciclo de vida de los individuos. La salida del hogar paterno, la formación de la pareja, el matrimonio, el divorcio, la formación de nuevas uniones, el nacimiento de los hijos... son transiciones que en muchas ocasiones vienen acompañadas con un cambio residencial, cambio que se produce básicamente a escala metropolitana. En este escenario, algunos de los cambios comportan una diferenciación entre centro y periferia: mientras que un primer hijo se asocia a un cambio reciente de vivienda hacia la periferia (Fiori et al., 2014) la emancipación o el divorcio normalmente utilizan el camino contrario (Rérat, 2012). En España, por ejemplo, se encuentra un mayor número de hogares unipersonales en los centros metropolitanos, mientras que las familias jóvenes migran a la periferia (López et al., 2013). El resultado es un aumento de la complejidad familiar en el interior de las zonas metropolitanas.

La movilidad residencial es el mecanismo por el cual las familias adaptan las preferencias habitacionales con las transiciones en el curso vital. Es por lo tanto una movilidad selectiva y como resultado especializa el espacio. Los centros envejecen más rápidamente mientras las periferias son jóvenes y acogen a familias con hijos (Mulder, 2006). Estos tipos familiares diferenciales producen comportamientos demográficos dispares, como es el caso de la fecundidad. Los centros urbanos, siguiendo el modelo tradicional, muestran menor fecundidad, y los municipios suburbanos una fecundidad más elevada (Kulu \& Vikat, 2007; Mulder, 2006).

En España, algunos de los centros metropolitanos muestran fecundidades más bajas que sus periferias, es el caso de Barcelona, San Sebastián, Cádiz, Sevilla o Madrid (Gil-Alonso et al., 2014), con diferencias observadas durante la década de los ochenta en Barcelona (Pujadas, 1992) o de los noventa en Madrid (Pozo y Rodríguez 2003). En cambio, en las provincias rurales, notablemente envejecidas, las zonas urbanas o grandes ciudades siguen siendo refugio de la mayor fecundidad. Si enfocamos la atención con mayor detalle en las áreas metropolitanas, encontraremos como las periferias no son homogéneas, y existen numerosas diferencias demográficas y sociales en ellas. Los niveles de fecundidad, entonces, dependen del tipo de municipio analizado (centro, periferia de crecimiento alto, periferia de crecimiento bajo, municipios de renta alta, de renta baja...), ya que cada uno de ellos se encuentra especializado en un tipo u otro de familia. La complejidad es la característica principal. Incluso los municipios con rentas mayores y menores pueden localizarse a muy poca distancia.

En este contexto, partimos de la hipótesis de trabajo de la existencia de una creciente diferenciación de la fecundidad metropolitana debido a los flujos migratorios internos. La llamada 
"movilidad residencial" filtraría hacia los municipios periféricos a las parejas jóvenes, en edad reproductiva, mientras que los centros urbanos más densos y las ciudades de la primera corona metropolitana serían lugar de asentamiento de otras formas familiares. El ciclo de vida de los individuos, y su plasmación territorial, marcarían las diferencias entre municipios. La hipótesis se sustenta, por un lado, en que en España buena parte de esta movilidad se relaciona directamente con la formación de la pareja (Módenes, 1998; Pujadas, 2009; Bayona y Pujadas, 2014), mientras que ciertas características de viviendas -aquellas más grandes, con jardín, o en un entorno menos densificado-, o simplemente aquellas viviendas económicamente accesibles (en un entorno donde la propiedad dominaba, tanto entre nacionales como de forma creciente para los extranjeros, Módenes et al., 2013), se encuentran únicamente localizadas en municipios periféricos. Para contrastar esta hipótesis, primero se presenta un análisis descriptivo de la evolución de la fecundidad en los municipios metropolitanos entre 1986 y 2012, y en segundo lugar se utiliza un análisis de regresión lineal para explicar las diferencias entre municipios. En este se consideran las migraciones internas como variable interpretativa, pero además se tiene en cuenta la inmigración internacional. En la primera década del siglo XXI en España, y en Cataluña en particular, ésta se configura como el principal elemento de la evolución demográfica del país, dando lugar a un escenario de fuerte crecimiento demográfico. Algunos municipios metropolitanos, en poco más de un lustro, pasan de porcentajes de población extranjera apenas significativos (por debajo del $5 \%$ ) a superar un $20 \%$ de la población total.

La inmigración extranjera tiene un impacto muy importante en la evolución metropolitana. En la primera década del siglo XXI se asientan alrededor de 750 mil personas en la RMB, con diferencias territoriales importantes. Primero se produce un asentamiento inicial en la ciudad de Barcelona, para una posterior dispersión metropolitana (Bayona \& Gil-Alonso, 2012). Las mujeres inmigrantes muestran fecundidades ligeramente más elevadas que el conjunto de la población en España y en Cataluña, con lo cual algunas de las diferencias internas podrían directamente atribuirse al distinto peso de las mujeres extranjeras. Devolder y Cabré (2009) analizan el impacto de la inmigración sobre el ISF español, y le atribuyen a ésta buena parte del aumento de la fecundidad. Existen, además de diferencias de intensidad, diferencias en el calendario, ya que la fecundidad de la población extranjera se produce en edades más jóvenes, tanto para España (Castro y Rosero-Bixby, 2011) como Cataluña (Devolder y Bueno, 2011).

Los datos observados en la ciudad de Barcelona contradicen, en parte, esta posible hipótesis (Pujadas et al., 2013). La presencia de un importante número de extranjeros en la ciudad de Barcelona no viene acompañada por aumentos en los niveles de fecundidad. A pesar del cambio poblacional (uno de cada cinco habitantes es actualmente nacido en el extranjero) la intensidad de la fecundidad no varía, y se mantiene inalterable y significativamente baja. Es necesario entonces analizar con mayor detalle la nacionalidad de los inmigrantes presentes.

\section{Estado de la cuestión}

La teoría de la transición demográfica, desde sus primeras formulaciones hasta las más recientes, ha destacado la importancia del proceso de urbanización para comprender los cambios del comportamiento reproductivo de las poblaciones urbanas. El descenso de la fecundidad estaría relacionado con el proceso de urbanización. Notestein (1953), uno de los primeros formuladores de la teoría, señala la influencia de la vida urbana sobre el cambio de mentalidad de la población, 
del trabajo en fábricas y del persistente aumento del coste de crianza de los hijos sobre los niveles de fecundidad. Poco después Coale y Hoover (1958) insisten en destacar la repercusión de la urbanización y de la industrialización en el declive de la natalidad. Como resultado de estas primeras reflexiones la tasa de urbanización pasó a ser uno de los factores explicativos del descenso de la fecundidad (además de la alfabetización, industrialización y descenso de la mortalidad) en el macro-estudio sobre el descenso de la fecundidad en Europa de la Universidad de Princeton finalizado en 1986 (European Fertility Project).

De modo que históricamente, la atención en el estudio de las diferencias en la fecundidad se ha centrado en la dualidad rural-urbano, a la que aún se sigue prestando atención. Áreas rurales más fecundas y centros urbanos con fecundidades más bajas caracterizan esta dualidad, con diferencias que a pesar de decrecer continuamente aún siguen manteniéndose (Kulu, 2013), aunque no siempre van en la misma dirección (Gil-Alonso et al., 2014). A pesar del gran interés histórico existente desde la geografía de la población por el estudio de las diferencias territoriales en la fecundidad (relacionadas con la transición demográfica y la difusión entre regiones del descenso de la fecundidad), actualmente se presta poca atención a este aspecto (Boyle, 2003), con contadas excepciones, a pesar que la localización territorial sigue teniendo un peso importante sobre las decisiones de curso de vida y de fecundidad.

Los planes de fecundidad se ven influenciados por ciertos aspectos del entorno donde las parejas residen. Las características de la vivienda, la distancia al centro (a veces en relación directa con la anterior), la existencia de transporte público, el tipo de barrio... son ejemplos de circunstancias estudiadas en un contexto norteamericano (Albrecht \& Albrecht, 2004). En Europa, existen también algunos estudios al respecto, como el de Courgeau (1989) donde analiza la formación de la familia en relación a la movilidad residencial, el de Fagnani (1991) donde relaciona el tamaño familiar con el del municipio de residencia en Francia, o los similares resultados de Brunetta y Rotondi (1991) para Italia, o Hank (2001) en Alemania. Los estudios recientes de Hill Kulu -y colaboradores- sobre los países nórdicos vuelven a situar el tema en primera línea de atención, y merecen ser comentados por separado, ya que en ellos se presentan varias hipótesis explicativas sobre las diferencias espaciales de la fecundidad en ámbitos metropolitanos (Kulu, 2013; Kulu \& Boyle, 2009). Estas se pueden resumir en tres líneas argumentales:

a) La hipótesis composicional: los niveles de fecundidad varían simplemente porque varía la composición de la población residente entre áreas. Es decir, la menor fecundidad en las ciudades debe atribuirse a las características socio-económicas de sus residentes, por ejemplo, la mayor proporción de población con estudios superiores, la mayor proporción de estudiantes, el menor porcentaje de casados o el mayor número de hogares unipersonales en relación a otras áreas.

b) La hipótesis contextual o comportamental: los factores ambientales y de entorno son de suma importancia para explicar las diferencias en la fecundidad. El entorno residencial o las condiciones de la vivienda son factores que adquieren alta representatividad. Por ejemplo, las áreas suburbanas con mayores espacios verdes y menor contaminación y densidad son reconocidas por varios progenitores como zonas preferibles en contraposición a las grandes ciudades. En estas mismas áreas es más fácil encontrar viviendas unifamiliares, con menor coste de la vivienda en relación con su superficie (Kulu \& Vikat, 2007). Las grandes ciudades, en cambio, el coste de los hijos es superior. 
En función del contexto, el comportamiento demográfico difiere por un mayor o menor costo de oportunidad.

c) La hipótesis de la selección de migrantes: las parejas urbanas que quieren tener un primer (o posterior) hijo deciden moverse a la periferia urbana ya que perciben que son áreas más apropiadas para la crianza, mientras aquellos sin planes reproductivos optan por seguir viviendo en la ciudad o trasladarse a ella (Kulu \& Vikat 2007, Milewski 2007).

Los resultados obtenidos en países nórdicos (Kulu \& Boyle, 2009; Kulu et al., 2009) indican la existencia de una menor fecundidad y producida en edades más avanzadas en los centros urbanos en comparación con los suburbios, o incluso con las áreas rurales. En general, los resultados muestran como los factores contextuales son más importantes que los composicionales para explicar las diferencias. En Inglaterra incluso se habla de la existencia de espacios geográficos caracterizados por culturas de alta (o baja) fecundidad (Fiori et al., 2014). Mientras las características sociodemográficas de las mujeres (educación y nivel de ingresos, básicamente) aportan poca explicación, las condiciones de la vivienda tienen mayor peso interpretativo (Kulu, 2013, Kulu \& Boyle, 2009). Por ejemplo, Kulu y Vikat (2007) encuentran que las familias que viven en viviendas unifamiliares tienen mayor fecundidad, y esta sobrerrepresentación explica parte de la mayor fecundidad suburbana o rural, en un resultado similar al obtenido por Mulder (2006), quien apunta que en las sociedades actuales, acceder a una vivienda adecuada parece ser una precondición a la formación familiar, y este requisito es menos difícil de conseguir en suburbios que en grandes ciudades. Como consecuencia, algunas parejas urbanas no llegan a alcanzar nunca su tamaño familiar ideal, por la dificultad de conseguir la vivienda necesaria. La proporción de parejas con fecundidad incompleta sería menor en áreas suburbanas y rurales, donde, al mismo tiempo, la familia deseada es de mayor tamaño que en zonas urbanas (Kulu, 2013).

En cuanto a la selectividad de los movimientos internos, el trabajo de Kulu y Boyle (2009) sobre la fecundidad suburbana en Finlandia, o el de Kulu et al. (2009) para cuatro países nórdicos, confirman la hipótesis de que los movimientos residenciales selectivos explican una parte significativa de los mayores niveles de fecundidad suburbana. Al mismo tiempo, parejas con la intención de tener hijos dejan la ciudad central para localizarse en los suburbios. Como consecuencia, aquellos residentes suburbanos que previamente residían en el centro metropolitano tienen mayor fecundidad que los residentes suburbanos no migrantes, a pesar de que entre estos últimos la fecundidad también es elevada (Kulu \& Boyle 2009).

En conclusión, la mayoría de estudios indican como la fecundidad suburbana es más alta que en los centros metropolitanos, donde además de una menor descendencia la maternidad se pospone notablemente, incluso cuando las características sociodemográficas de las madres son estadísticamente controladas las diferencias se mantienen. De esta forma, las principales causas de la mayor fecundidad suburbana deben atribuirse tanto a factores contextuales como a la migración selectiva de parejas.

\section{Marco geográfico, datos estadísticos y metodología de análisis}

El ámbito de análisis utilizado en este trabajo es el metropolitano, y el caso de estudio es la Región Metropolitana de Barcelona (RMB), la segunda de mayor tamaño poblacional en España 
después de la capital y su metrópolis, Madrid. Esta comprende una superficie de $3.236 \mathrm{Km}^{2}$ y engloba un conjunto de 164 municipios, agrupados en siete comarcas. Era también uno de los siete ámbitos definidos en el Pla Territorial de Catalunya, y tiene su formulación original en los años sesenta (Esteban, 2003), configurándose en numerosos estudios como la unidad de análisis metropolitana preferente. Tiene una población justo por encima de los cinco millones de habitantes (5.051.502 a enero de 2013, con datos del Padrón continuo de población), y una configuración de carácter policéntrico (Roca et al., 2011). La capital, Barcelona, contiene un tercio de la población total de la metrópolis, y se encuentra inmersa en un continuado proceso de suburbanización desde los años setenta. La RMB cuenta también con seis ciudades que superan los cien mil residentes, un largo número de municipios por encima de los diez mil residentes, y aún un número importante de municipios que conservan un carácter menos urbano (por no decir rural), por debajo de este umbral poblacional. La RMB tiene también un prolongado historial de construcción metropolitana, con diferencias socioeconómicas entre municipios de importancia (ver Pujadas et al., 2007, Rubiales et al., 2012; Costa \& Porcel, 2013), que se encuentran en la base de la diferenciación de los comportamientos demográficos.

Para el estudio de la fecundidad a nivel municipal se han utilizado los datos de los nacimientos por edad de la madre y lugar de residencia proporcionados por el Institut d'Estadística de Catalunya, con una serie que tiene su origen en el año 1986, con 2012 como última fecha disponible. La población de referencia, las mujeres entre 15 y 49 años por edad, necesaria para el cálculo del Índice Sintético de Fecundidad (ISF) ${ }^{6}$, se ha obtenido del mismo servidor estadístico, utilizando las estimaciones intercensales y postcensales de población. Se ha empleado las estimaciones de población debido a que estas se aproximan mejor al cálculo de la población extranjera en comparación al Padrón continuo (que las sobreestima), y porqué es la población de referencia al calcular el ISF para el conjunto catalán o español por parte de los institutos de estadística (las diferencias, en un contexto de muy baja fecundidad, creemos que son importantes). Tiene, en cambio, el problema de que únicamente se encuentran disponibles para los municipios de más de 45 mil habitantes, con lo cual contamos con 19 municipios en la RMB por encima de este umbral. Como dicha información también se publica a nivel comarcal, se ha podido reconstruir siete "restos comarcales", con lo cual contamos con 26 unidades de análisis (Figura No 1). Un segundo problema caracteriza los datos, como es la imposibilidad de desagregación por nacionalidad, lo que nos impide una aproximación con mayor detalle.

El análisis descriptivo ha sido completado por un modelo de regresión lineal, donde la variable dependiente analizada ha sido el Índice Sintético de Fecundidad (ISF) de las 26 unidades territoriales (ciudades y restos comarcales) estudiadas. Los niveles de fecundidad serán explicados a partir de siete variables independientes que representan las tres dimensiones que consideramos interpretativas de las diferencias territoriales en las pautas de fecundidad: 1) las tasas de migración neta interna, calculadas de forma diferencial para la población española y la población extranjera (ya que no se dirigen a los mismos municipios), y que nos informa de la intensidad de los cambios migratorios internos entre municipios de la propia RMB; 2) el porcentaje que representan los diferentes grupos inmigrantes en cada uno de los municipios, diferenciando por origen continental de la nacionalidad; y 3 ) el valor de la renta familiar disponible de cada uno de los municipios analizados. Estos parámetros se encuentran resumidos en el Cuadro № 1.

También conocida como Tasa de Fecundidad Global (TFG), Total Fertility Rate (TFR) en inglés. Expresa el número medio de hijos por mujer en edad fértil. 
Figura No 1

Localización de las ciudades de más de 45.000 habitantes y "restos comarcales",

Región Metropolitana de Barcelona

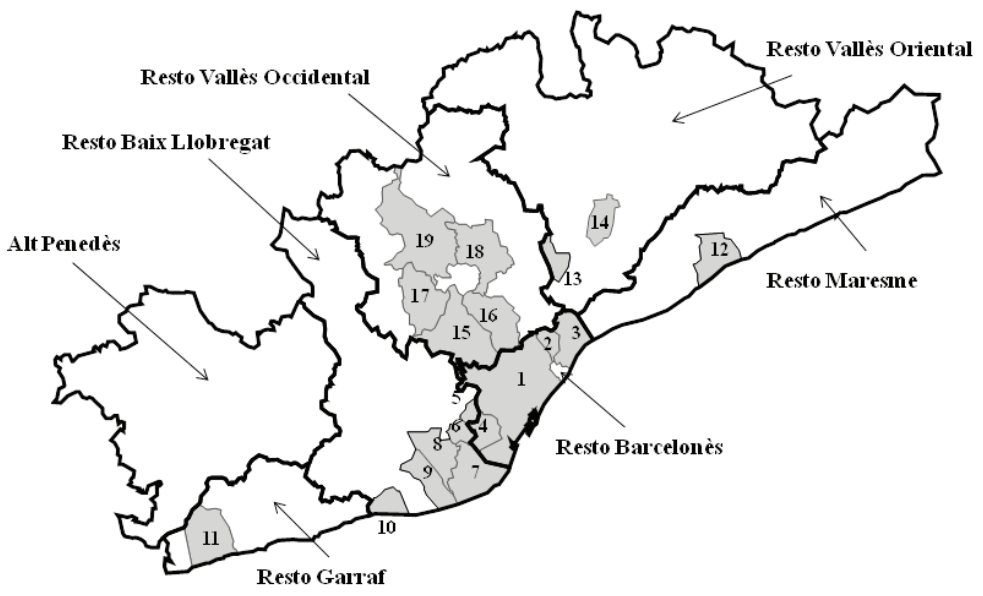

\begin{tabular}{|l|l|l|l|l|l|l|l|}
\hline 1. & Barcelona & 6. & Cornellà de Llobregat & 11. & Vilanova i la Geltrú & 16. & Cerdanyola del Vallès \\
\hline 2. & Sta. Coloma de Gramenet & 7. & El Prat de Llobregat & 12. & Mataró & 17. & Rubí \\
\hline 3. & Badalona & 8. & Sant Boi de Llobregat & 13. & Mollet del Vallès & 18. & Sabadell \\
\hline 4. & L'Hospitalet de Llobregat & 9. & Viladecans & 14. & Granollers & 19. & Terrassa \\
\hline 5. & Esplugues de Llobregat & 10. & Castelldefels & 15. & Sant Cugat del Vallès & & \\
\hline
\end{tabular}

Fuente: Elaboración propia.

Temporalmente se compara el inicio de la primera década del siglo XXI con el final de esta (2001 y 2009). En el primer caso, consideramos que el efecto de la inmigración internacional es aún escaso. En el segundo, encontramos los niveles más altos de fecundidad, previos a su descenso producto de la crisis. No se ha escogido un año más reciente (los datos alcanzan a 2012) debido a la situación de inestabilidad que conlleva la crisis, donde, por ejemplo, se han desdibujado las pautas de movilidad interna preexistentes y la situación puede caracterizarse por la indefinición (Pujadas et al., 2016). Después de comprobar posibles correlaciones entre los datos de 2001 y 2009, se han desarrollado seis modelos de regresión lineal. Tres de ellos consideran el año 2001, los otros tres son para 2009. Cada uno de los dos grupos de modelos incluye: 1) un modelo "urbano" que emplea todas las variables independientes pero solo para los municipios de más de 45 mil habitantes; un modelo "general" donde se utilizan también todas las variables pero en este caso se emplean todas las unidades territoriales; y finalmente, 3 ) un nuevo modelo "general" para las 26 unidades espaciales donde únicamente se añaden las variables que son estadísticamente significativas. En consecuencia, resultan dos grupos de modelos en las comparaciones entre 2001 y 2009 , unos para toda la RMB, otros solo para los municipios de más de 45 mil habitantes. 
Cuadro No 1

Variables utilizadas en el análisis de regresión

\begin{tabular}{|l|l|l|}
\hline \multicolumn{2}{|c|}{2001} & \multicolumn{1}{|c|}{ Variables } \\
\hline Dimensiones & \multicolumn{1}{|c|}{2009} \\
\hline Fecundidad & Índice Sintético de Fecundidad & Índice Sintético de Fecundidad \\
\hline Renta & Renta familiar disponible por municipio & Renta familiar disponible por municipio \\
\hline $\begin{array}{l}\text { Cambios } \\
\text { residenciales }\end{array}$ & $\begin{array}{l}\text { Tasas de migración neta de extranjeros } \\
\text { Tasas de migración neta de españoles }\end{array}$ & $\begin{array}{l}\text { Tasas de migración neta de extranjeros } \\
\text { Tasas de migración neta de españoles }\end{array}$ \\
\hline $\begin{array}{l}\text { inmigración } \\
\text { extranjera }\end{array}$ & $\begin{array}{l}\% \text { europeos } \\
\% \text { americanos } \\
\% \text { africanos }\end{array}$ & $\begin{array}{l}\% \text { europeos } \\
\% \text { americanos } \\
\% \text { asiáticos } \\
\% \text { africanos }\end{array}$ \\
\hline
\end{tabular}

Los municipios de menos de 45 mil habitantes son agrupados en "restos comarcales". Entre 2001 y 2009 se añaden dos nuevos municipios a la muestra.

El Movimiento Natural de la Población (MNP) recoge la dinámica demográfica, es decir, nacimientos y defunciones totales y por edades. El Padrón continuo de Población es el registro local de población, referenciado a 1 de enero de cada año, y que recoge el stock de población de un municipio (con independencia de su situación legal). La Estadística de Variaciones Residenciales registra los cambios de municipio de residencia, dando lugar a estadísticas de inmigrantes y emigrantes según sus características demográficas (sexo, edad, nacionalidad y país de nacimiento).

Fuentes: Elaboración propia basado en Movimiento Natural de la Población (MNP), 2001 y 2009; Censo de 2001, Padrón continuo de 2009, Estadística de Variaciones Residenciales (2001-2009), todas ellas con datos del INE, y estimaciones municipales de población (con datos de Idescat).

\section{Análisis descriptivo de la evolución de la fecundidad en los municipios de la RMB}

\section{La evolución de la fecundidad en Cataluña y España}

La fecundidad metropolitana de estos últimos años se sitúa en un contexto español y catalán caracterizado por una muy baja fecundidad, aunque desde unos años atrás se vienen observando ligeros signos de recuperación. El descenso de la fecundidad española se produce relativamente tarde, en comparación con otros países de Europa. Aún en 1975 el ISF español se sitúa entre los más altos de Europa, con 2,8 hijos por mujer, bastante por encima de los 1,9 de Francia, los 1,8 de Gran Bretaña o incluso el 2,2 de Italia. Desde entonces, en cambio, la caída de la fecundidad es prolongada y puede ser caracterizada por su fuerte intensidad, ya que los valores de la fecundidad española que se registran en los años noventa (1,15 hijos por mujer de 1998) se sitúan prácticamente en mínimos mundiales. Desde estos mínimos, la recuperación posterior es más lenta, y se sustenta en un ciclo económico expansivo y en la aportación de la inmigración internacional. Diez años más tarde, en 2008, se llegan a alcanzar los 1,44 hijos por mujer, que sigue siendo una fecundidad 
baja a escala europea, para descender posteriormente al llegar la crisis económica. De esta forma, en 2012 la fecundidad española vuelve a situarse en valores bajos (1,32 hijos por mujer), lejos del 1,58 del conjunto de países de la Unión Europea. Entre los países comunitarios únicamente Polonia $(1,30)$ y Portugal $(1,28)$ muestran menor fecundidad, mientras Eslovaquia, Hungría, Grecia (todos ellos con 1,34) o Alemania $(1,38)$ o Chipre $(1,39)$ mantienen fecundidades justo por encima. Lejos quedan países como Francia o Irlanda (en ambos casos 2,01), o Gran Bretaña $(1,92)$ o Suecia $(1,91)$.

La tendencia observada en Catalunya o en la provincia de Barcelona es similar a la del conjunto español, con mínimas diferencias en la intensidad y en el calendario de los cambios. En la actualidad, y con datos provisionales para el año 2013, en Cataluña se observa una fecundidad de 1,34 hijos por mujer (por 1,27 en el conjunto español), que se sustenta en una fecundidad similar de las mujeres autóctonas (1,25 en Cataluña por 1,23 en España) y ligeramente superior de las mujeres extranjeras $(1,69$ por 1,52), además del mayor peso que estas adquieren sobre el total de población catalana. Si la crisis económica se dejó notar poco en un primer momento sobre la natalidad en Catalunya, los efectos acumulados llevan los nacimientos a descender desde los 89 mil de 2008 a los 71.493 de 2013.

\section{Las dinámicas demográficas recientes: suburbanización e inmigración extranjera}

La ciudad de Barcelona alcanza su máximo de población en la década de los ochenta (1.752.627 habitantes en 1981). A pesar de ello, la ciudad empezaba ya durante los setenta a perder población por migración interna, ya que el crecimiento únicamente se sustentaba en el saldo natural positivo (Domingo et al., 2004). La saturación de la ciudad central (de apenas $100 \mathrm{~km}^{2}$ ), y el descenso del tamaño medio del hogar son los responsables de esta dinámica. Desde entonces y hasta la irrupción de la inmigración internacional, Barcelona pierde población, situándose justo por debajo del millón y medio de residentes. La intensidad del fenómeno migratorio internacional produce una fuerte recuperación del número de habitantes, hasta un máximo de 1.621 .537 habitantes, un $17,5 \%$ extranjeros (y más del $20 \%$ si el criterio es el país de nacimiento). Desde entonces, y a consecuencia de la crisis, la evolución es más errática, estancándose de nuevo la población y entrando en un lento declive, con 1.611 .822 residentes en enero de 2013, manteniéndose el porcentaje de extranjeros $(17,4 \%)$.

La dinámica del conjunto metropolitano se caracteriza, en cambio, por un estancamiento de la población entre 1981 y 1996 alrededor de los 4,2 millones de habitantes, siendo el único flujo importante el que se producía entre centro y periferia. La llegada de inmigrantes internacionales produce, de forma similar al centro, un fuerte crecimiento de la población, que supera en la actualidad los cinco millones de habitantes, un $14,5 \%$ de ellos extranjeros. En este contexto, el peso del municipio central es cada vez menor, alrededor del $32 \%$ en 2013 , cuando en los años setenta se aproximaba al $50 \%$.

La reciente inmigración internacional reproduce el proceso territorial vivido en décadas precedentes. Después de una primera inserción en Barcelona, que funciona como puerta de entrada de los flujos migratorios internacionales (Domingo et al., 2004), existe un proceso de desconcentración, hacia la periferia. A diferencia de los españoles, que se instalan en la segunda corona, los extranjeros se localizan en los municipios adyacentes, como L'Hospitalet de Llobregat o Santa Coloma de Gramenet, donde, representan el 23\% de la población (Figura № 2). Se trata de 
Figura No 2

Proporción de población extranjera, por agregado continental, sobre la población total, RMB, 2010
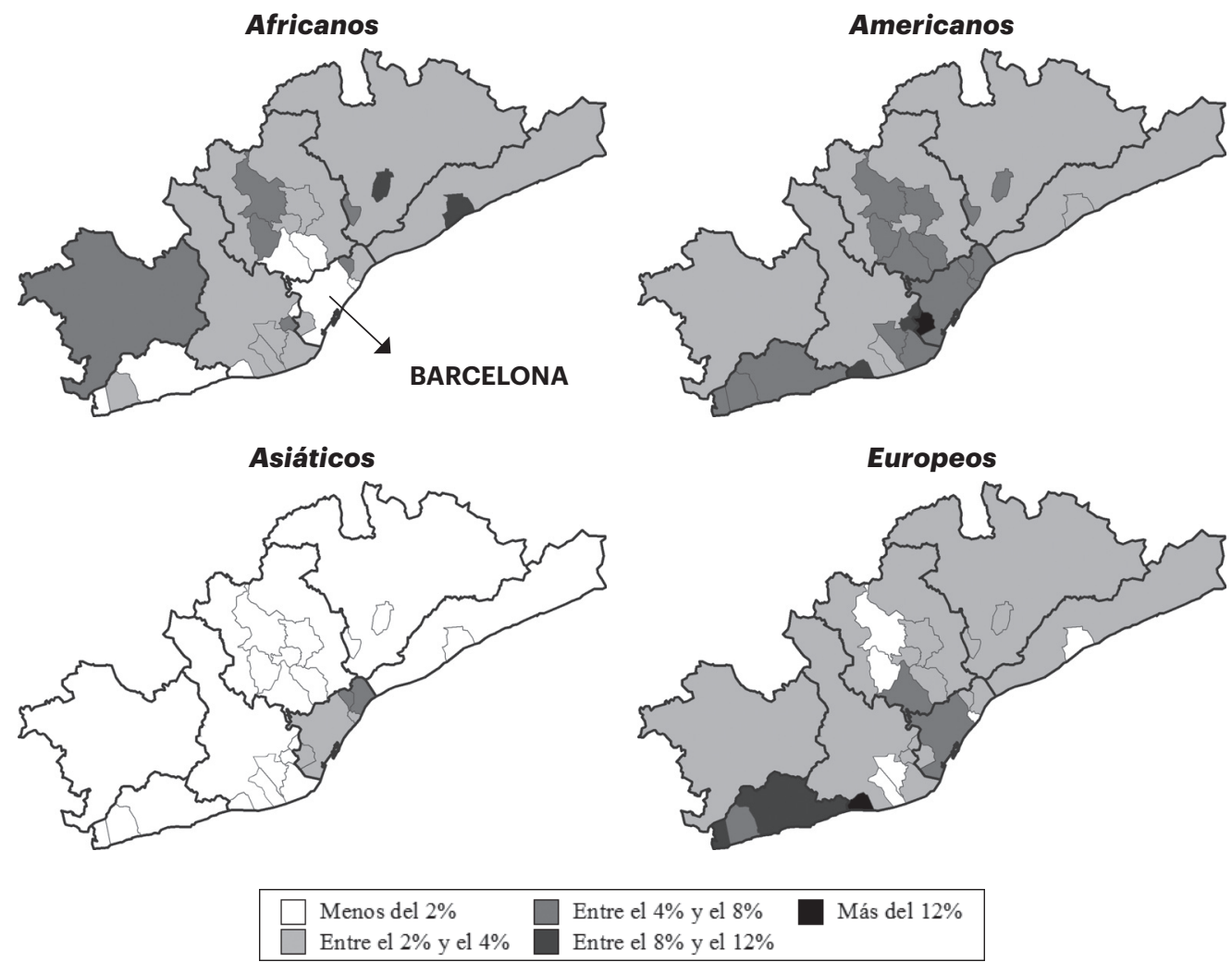

Fuente: Padrón de población de 2010, con datos del INE.

municipios de la primera corona metropolitana que crecieron a remolque de antiguos procesos de industrialización e inmigración interna en España, ya en la década de 1920 en el primer caso, pero especialmente durante las décadas de 1960 y 1970. Dos procesos facilitan la entrada de inmigrantes extranjeros, por un lado, y con el paso del tiempo, el proceso de envejecimiento, que domina algunos de los barrios de estos municipios. Por el otro, las migraciones internas hacia la periferia de la población española procedente no solo en la ciudad de Barcelona, sino también de los municipios más densos de la primera corona metropolitana ${ }^{7}$ (Figura № 3). En ellos se asientan inmigrantes extranjeros, que encuentran vivienda barata, próxima a Barcelona y bien conectada con ésta por transporte público. Sin embargo, las pautas de localización difieren por origen continental. Mientras los asiáticos experimentan altas concentraciones en Barcelona y en algún municipio de la primera corona, los africanos se encuentran en la periferia, con cierto grado de concentración. Americanos y Europeos generalmente se encuentran más dispersos (Galeano et

La migración neta es la diferencia entre inmigrantes y emigrants. Está expressada como tasa, es decir, en relación a la población media del período y en formato anual. 
Figura $\mathrm{N}^{\circ} 3$

Tasa de migración interna neta por nacionalidad, RMB, 2001-2009

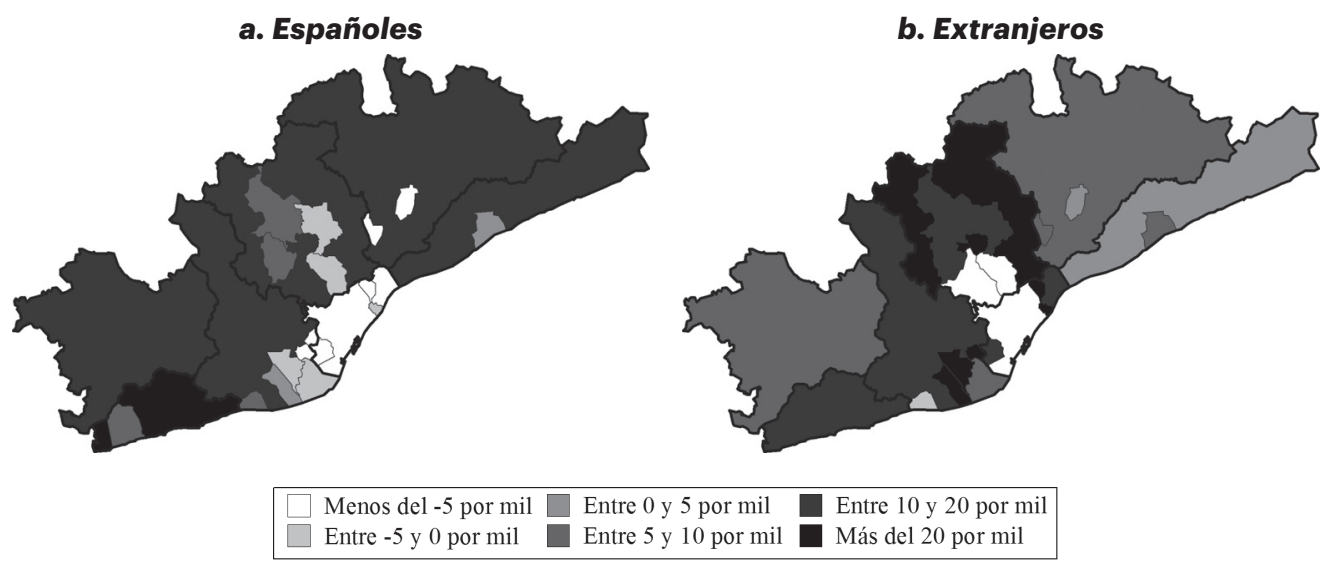

Fuente: Estadística de Variaciones Residenciales, 2001-2009, con datos del INE.

al., 2014), aunque con una leve concentración en Barcelona y las ciudades más grandes (americanos) o en los municipios de renta más elevada (europeos).

Los fuertes flujos internos de suburbanización han producido la desconcentración metropolitana (Pujadas et al., 2016), acompañados por la redistribución de la actividad económica y de los lugares de trabajo (Alberich, 2010). La inmigración internacional, que primero se asienta en el centro, más tarde reproduce las migraciones internas metropolitanas, dirigiéndose a los municipios de la primera corona metropolitana, precisamente aquellos mismos que acogieron en los años sesenta y setenta, años de fuerte migración campo-ciudad en España. En estos flujos existe un proceso de selección, tanto demográfico como social, y el resultado nos indica la pérdida de parejas jóvenes, alrededor de los 25-35 años de edad, pero también de las clases sociales más populares y la población con menor nivel de estudios. No es que Barcelona presente tasas de expulsión más elevadas que otros municipios, lo que sucede es que el centro metropolitano carece de capacidad de atracción, con tasas de inmigración metropolitanas realmente bajas.

\section{Análisis descriptivo: la evolución de la fecundidad en la RMB, 1986-2012}

La fecundidad metropolitana se comporta de forma similar a la del conjunto de Catalunya, aunque en valores ligeramente por debajo. En 1986, año inicial de análisis, el ISF de la RMB era de 1,37 hijos por mujer, similar al 1,39 de Catalunya. Desde entonces evolucionan de forma paralela, con mayor crecimiento en el conjunto catalán, como lo demuestran los valores de 2008 (1,47 en la RMB para 1,58 en Catalunya) o del reciente año 2012, último con datos disponibles (1,34 en la RMB y 1,43 en Catalunya). Esta fecundidad metropolitana esconde una amplia diversidad local, que se intensifica en fases de mayor fecundidad. Por un lado, la ciudad de Barcelona muestra unos ISF particularmente bajos, alrededor de 1,2 hijos por mujer durante los últimos 25 años, aunque no siempre representa los valores mínimos. 
En épocas de descenso de la fecundidad algunas ciudades de la primera corona llegan a presentar fecundidades con un ISF por debajo de 0,9. Por el otro los máximos llegan a superar el 1,85. Además de la intensidad, la fecundidad se caracteriza por un calendario tardío y una edad media a la maternidad por encima de los 30 años (31,4 en 2010), con un crecimiento de la fecundidad extramatrimonial (un tercio de los niños lo son de madres no casadas). Este último indicador crece progresivamente desde valores muy bajos (7,2\% en 1986), ejemplo de la extensión de la Segunda Transición Demográfica. Ambos fenómenos son más importantes en la ciudad central, aunque se expanden rápidamente por la región metropolitana.

La estabilidad de la fecundidad de la ciudad de Barcelona puede observarse en la Figura No 4, donde se muestra también la evolución del ISF en otras seis ciudades metropolitanas, todas ellas por encima de los cien mil residentes, y del conjunto catalán. En 1986 la fecundidad es descendente, como continuación de un proceso de disminución desde los altos valores de los años setenta hasta alcanzar los mínimos de mediados de los años noventa. En cambio, la ciudad de Barcelona no se ve afectada por las variaciones generales y se mantiene siempre alrededor de los 1,20 hijos por mujer. Dos de las ciudades vecinas de Barcelona, Santa Coloma de Gramenet y L'Hospitalet de Llobregat, caracterizadas por albergar población de rentas bajas (que en la mayoría son nacidos fuera de Cataluña), experimentan las mayores variaciones, tanto en las épocas de menor fecundidad (donde esta experimenta los valores mínimos, es el caso del 0,87 hijos por mujer de l'Hospitalet en 1995) como en las de crecimiento de esta. Si la menor fecundidad puede explicarse por la acentuación de los mismos procesos que se dan en Barcelona ciudad (pérdida de jóvenes a la periferia y envejecimiento de la población) en cambio es más difícil entrar a explicar las diferencias en los valores máximos de fecundidad, ya que estos se deben a la desigual composición por nacionalidades de los extranjeros allí residentes. Ambas ciudades experimentan crecimientos importantes de su fecundidad en épocas de crecimiento económico, de forma similar a Catalunya, pero varia el nivel alcanzado En Santa Coloma de Gramenet se llega a un máximo de 1,75 hijos por mujer en 2008, por solo 1,36 en L'Hospitalet de Llobregat. En los dos casos, el porcentaje de residentes extranjeros es similar (en la actualidad por encima del $20 \%$ y más importante que en Barcelona), pero no su composición. Mientras en Santa Coloma de Gramenet dominan los asiáticos (chinos y pakistaníes) y los africanos (marroquíes), en l'Hospitalet de Llobregat las mujeres son latinoamericanas. La mayor fecundidad de unas (africanas y asiáticas) respecto las otras (latinoamericanas) explica la diferente intensidad alcanzada ${ }^{8}$.

En último lugar, falta analizar que sucede en las ciudades metropolitanas situadas en la segunda corona, más alejadas del centro, como son los casos de Mataró, Terrassa, Sabadell, pero también de Badalona, más cercana a Barcelona. En los cuatro casos, y para todos los años, la fecundidad sigue la dinámica general, con crecimientos en épocas de bonanza y fuertes descensos en épocas de crisis. Se caracterizan por mostrar valores superiores al conjunto catalán, especialmente los casos de Terrassa y Mataró, ciudades con un fuerte crecimiento durante los últimos años vía inmigración interna. Los máximos de 1,85 en Terrassa o 1,77 en Mataró en 2008

El peso de la inmigración internacional en el conjunto de la fecundidad se entiende rápidamente observando que sucede en los nacimientos. En 2010, el 24,1\% de los 486.575 niños nacidos en España tenía un progenitor extranjero, porcentaje que en Cataluña alcanza a uno de cada tres nacimientos (33,7\%). Mayores diferencias se observan a nivel local. En 2010, el porcentaje de nacimientos de madres extranjeras oscila entre el 13,5\% de Viladecans al 46,7\% de l'Hospitalet de Llobregat, mientras en Barcleona es del 30,8\%. En general, aquellos municipios con mayor proporción de nacimientos de madres extranjeras son los mismos que tienen mayor fecundidad, a excepción de la ciudad central. 
Figura No 4

Evolución de la fecundidad (ISF) en los siete municipios de mayor tamaño de la RMB, 1986-2012

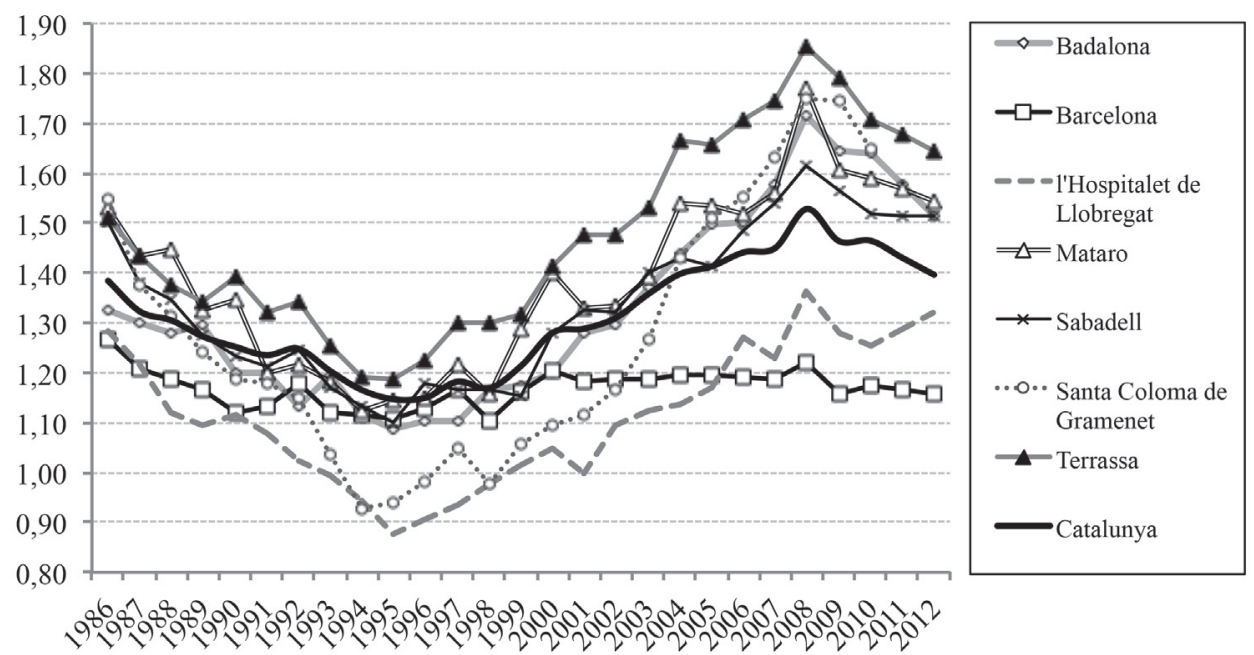

Fuente: Elaboración propia basado en Movimiento Natural de la Población y estimaciones de población intercensales y postcensales del Idescat.

así lo demuestran. Además, y para reforzar dicha dinámica, la población extranjera presente en estas ciudades es predominantemente africana.

Una de las mejores maneras de observar la dispersión es la comparación entre los valores máximos y mínimos de la fecundidad en un año determinado. Si a mediados de los años ochenta, en pleno proceso de descenso de la fecundidad, entre el mínimo de Barcelona $(1,27)$ y el máximo de Santa Coloma de Gramenet $(1,55)$ existía una distancia de 0,28 puntos, que se mantiene e incluso decrece en los años de menor fecundidad, esta diferenciación se expande a los 0,63 puntos de 2008, del mínimo del 1,22 de Barcelona al máximo de 1,85 de Terrassa, rango que se mantiene elevado en 2012 (entre los valores extremos de 1,16 de Barcelona y 1,65 de Terrassa) a pesar del descenso general de la fecundidad. Es decir, que en 25 años se dobla el diferencial de fecundidad entre las ciudades metropolitanos más y menos fecundas.

Los mapas de la Figura No 5 muestran que en 1986 la mayor fecundidad se producía en las áreas costeras suburbanas. Diez años más tarde, y en el momento de menor fecundidad, es en Sant Cugat del Vallès donde se alcanza la máxima fecundidad (1,47 hijos por mujer en 1996 y 1,67 en 2001). Se trata de un municipio de renta media elevada y que ha experimentado un crecimiento importante a lo largo del periodo analizado (de 35 mil habitantes en 1986 a 83 mil en 2001 y 86 mil en 2013) debido sobre todo a la llegada de inmigrantes internos españoles de renta media-alta. Sin embargo, en 2009, pero también en 2012, e influidos por la llegada de inmigrantes extranjeros, cambia completamente la distribución de la fecundidad. Son ciudades suburbanas como Terrassa (1,79 hijos por mujer en 2009) las que presentan mayor fecundidad, seguida por la comarca del Vallès Oriental (en 2009) o del Alt Penedès (en 2012), de carácter más rural. Son áreas periféricas que experimentan altos crecimientos internos por suburbanización. Así, 
Figura No 5

Evolución reciente del Índice Sintético de Fecundidad (ISF) en la RMB, 1996-2010
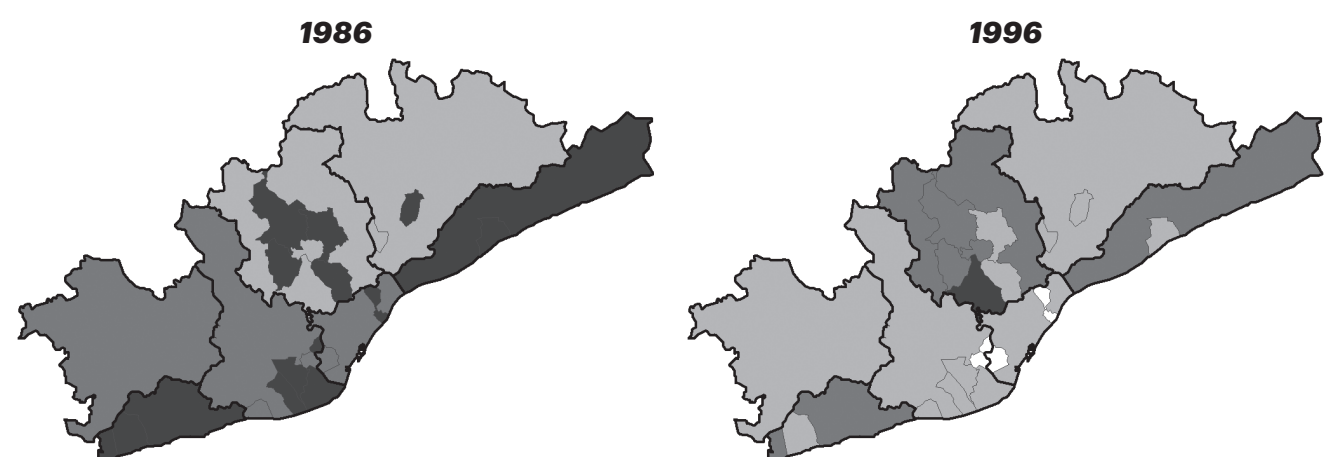

2001

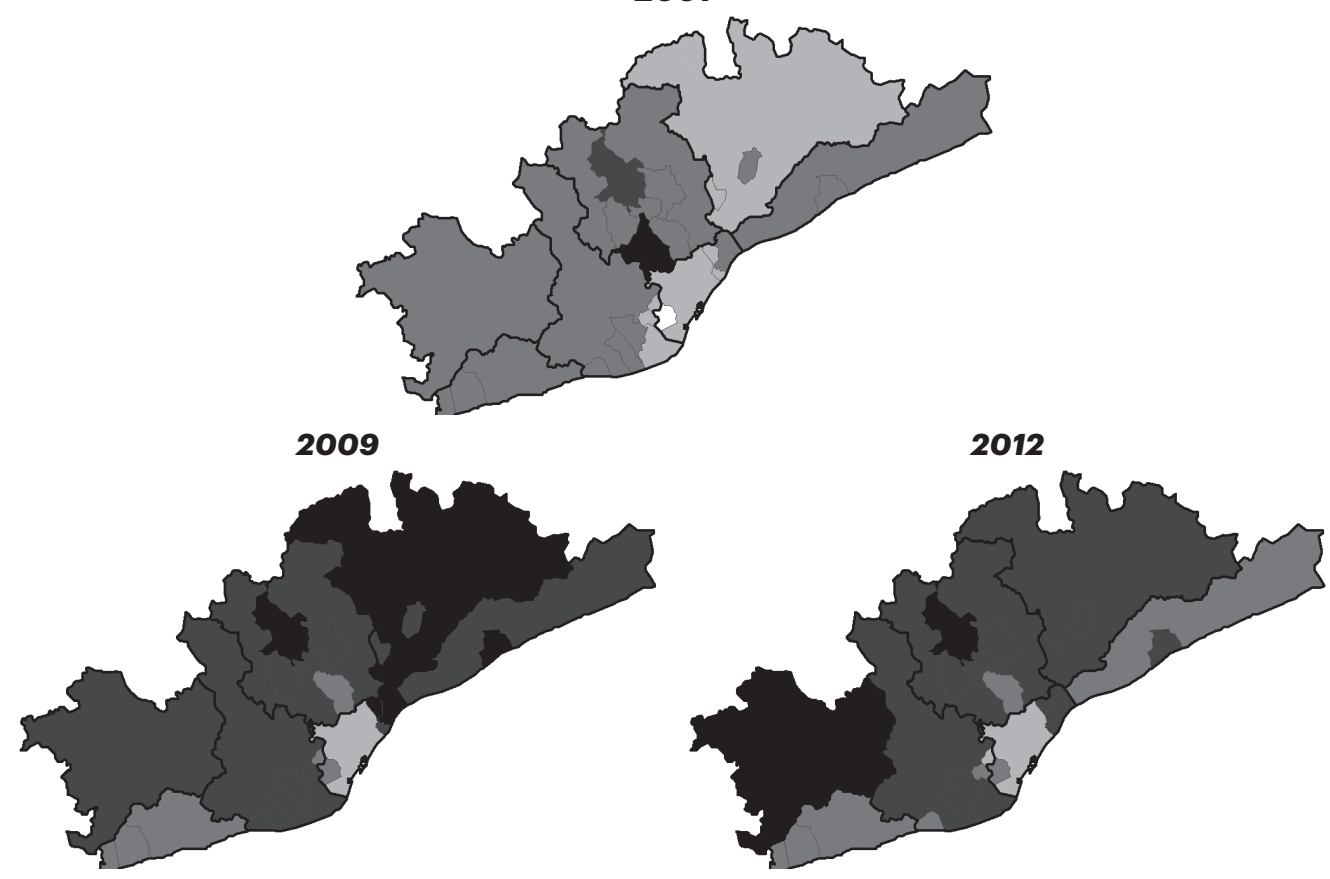

Menos de 1,00 hijo por mujer $\square$ Entre 1,01 y 1,20 hijos

Entre 1,21 y 1,40

Entre 1,41 y 1,60

Más de 1,60

Fuente: Elaboración propia basado en Movimiento Natural de la Población y estimaciones de población intercensales y postcensales del Idescat.

los municipios menores acogen parejas jóvenes en viviendas unifamiliares, mientras que algunas grandes ciudades periféricas siguen creciendo al aprovechar viejos espacios industriales internos reconvertidos a nuevo suelo residencial, alojando a personas que buscan en la periferia viviendas (en este caso plurifamiliares) a precios más asequibles en los años de la burbuja inmobiliaria. Además, estos municipios de mayor fecundidad coinciden con la distribución de africanos, pero también de asiáticos (es el caso de las ciudades de la primera corona metropolitana del este de Barcelona, como Badalona o Santa Coloma de Gramenet). 
En el lado opuesto, las zonas con valores de fecundidad menores pasan de encontrarse en los municipios más lejanos en 1986 a los de clase trabajadora de la primera corona metropolitana como L'Hospitalet de Llobregat (en 1996 un mínimo de 0,91 hijos por mujer), Cerdanyola o Esplugues. Estos dos últimos, en la actualidad, presentan los valores más bajos junto a Barcelona, y se caracterizan por un peso poco importante de los extranjeros.

En definitiva, el análisis descriptivo sugiere que los factores explicativos más importantes de la fecundidad experimentan cambios importantes a principios y finales de la década. Hasta 2001 parece que existe una relación entre nivel de riqueza del municipio y fecundidad, pues son municipios como Sant Cugat de Vallès o aquéllos situados en los dos corredores litorales, de mayor renta media, los que reciben parejas jóvenes de clase media y media-alta con intención de formar nuevas familias. Más recientemente, en cambio, la proporción de extranjeros parece adquirir mayor notoriedad. El análisis descriptivo no nos permite sin embargo alcanzar conclusiones más sólidas, y por lo tanto se emplean a continuación modelos de regresión lineal con la intención de obtener una constancia estadísticamente significativa de estos hechos.

\section{Análisis estadístico de las variables que influyen en la fecundidad metropolitana}

Para contrastar nuestras hipótesis, se ha utilizado un análisis de regresión, con la intención de identificar aquellas variables que intervienen en diferenciar los municipios en función de su fecundidad. Se emplean dos años de análisis, a principios y final de la primera década de siglo XXI. El año 2001, que coincide con el momento inicial de crecimiento de la inmigración internacional y que puede considerarse como ejemplo de las pautas de fecundidad precedentes al momento del boom migratorio; y el año 2009, año caracterizado por presentar la máxima intensidad de la fecundidad, a pesar de haberse ya iniciado la crisis económica y donde algunas de las variables interpretativas empleadas se encuentran en el punto de máxima diferenciación.

Antes de presentar los resultados, son necesarias algunas consideraciones metodológicas acerca de algunos problemas encontrados en el análisis: a) la existencia de pocos casos de observación; b) la heterogeneidad de los municipios analizados; y c) la posible existencia de autocorrelación.

a) La variable dependiente del modelo es el Índice Sintético de Fecundidad, indicador transversal que nos calcula la intensidad de la fecundidad. Este únicamente se encuentra calculado para los municipios que cuentan con más de 45 mil habitantes, lo que nos produce no muchos casos de observación. Para contrarrestar este efecto, se ha agrupado el resto de municipios en siete grupos o "restos comarcales", lo que agranda el tamaño muestral. A pesar de ello, seguimos contando con un número reducido de observaciones, con lo que es más difícil obtener modelos válidos y con significatividad estadística, y que estos se encuentran, además, afectados en mayor medida por casos atípicos. Es por esta razón que en el modelo para el año 2009 un municipio ha sido excluido, Sant Cugat del Vallès, ya que su comportamiento difería del resto de municipios analizados.

b) Los contrastes entre municipios de una región metropolitana son muy marcados, debido a su morfología diversa, al desigual tamaño, a su composición interna y a su evolución histórica. En algunos casos se encuentran pautas regulares de fecundidad en fun- 
ción de la nacionalidad o el nivel de ingresos. Si embargo, estas diferencias tampoco se mantienen constantes en todas las areas analizadas ya que algunas características específicas de los municipios, como la disponibilidad de vivienda o la renta media del municipio pueden también alterar las pautas de fecundidad de los subgrupos.

c) La autocorrelación espacial es un problema que se observa comúnmente en los análisis multivariables cuando éstos se aplican al análisis del territorio (Harvey 2010). A pesar de que los datos no muestran problema alguno de colinealidad entre variables (ANOVA tests entre 0,000 y 0,003), si que es cierto que los resultados obtenidos para algunos grupos nacionales están potenciados por una coincidencia con una alta o baja fecundidad de los españoles asociada a condicionantes territoriales, por un efecto difusión. Es decir, la importancia explicativa para la fecundidad general que tiene la presencia de población inmigrante de un determinado origen nacional se puede potenciar o reducir en función de la existencia de un patrón de fecundidad sistemático entre sus convecinos autóctonos. Dos casos ejemplifican esta situación: la relación inversa entre población de nacionalidad americana y la fecundidad en 2001, y la fuerte relación entre fecundidad y población africana en 2009. En 2001 la población americana se concentra en grandes ciudades, especialmente Barcelona. Esta población presenta un patrón de fecundidad con bajas tasas, porque se tienen los hijos a edades tempranas, frecuentemente antes de su proyecto migratorio. Y por otro lado una mayor implantación en municipios densos y centrales, dónde la fecundidad de la población española menor que en el resto del territorio. Como resultado, la relación inversa entre el porcentaje de población de origen americano y la fecundidad del municipio (coeficiente beta estandarizado de $-0,42)$ no solo se debe al comportamiento reproductivo general de la población americana en la RMB, sino también al comportamiento reproductivo de la población española de los municipios en los que se localiza. El segundo caso, la contribución de la población africana en 2009 a las tasas de fecundidad sigue una lógica similar. Al contrario del caso anterior, la fecundidad de 2009 y la población africana se encuentran positivamente relacionadas $(0,44)$. La fecundidad de las africanas es sistemáticamente más alta que el resto de grupos (con excepción de las asiáticas, con poca representatividad), y además generalmente residen en municipios periféricos de baja extracción social. Se da la coincidencia que en bastantes ocasiones se trata de los mismos municipios que acogen migrantes internos, jóvenes familias autóctonas en búsqueda de un entorno y una vivienda más agradables a sus necesidades (o a un precio menor, en años de boom inmobiliario), y donde satisfacer sus deseos de reproducción. Por lo tanto, el coeficiente estandarizado no solo informa del comportamiento reproductivo de los africanos, sino también el de los vecinos españoles.

Estos tres problemas nos obligan a interpretar los resultados con cierta cautela, y justifican la necesidad del análisis descriptivo utilizado previamente. Sin embargo, la regresión lineal seguiría siendo un buen instrumento para resumir la información y valorar el peso diferencial de las variables independientes sobre la variable dependiente.

Los resultados de la regresión (Cuadro No 2) muestran que entre los años 2001 y 2009 los determinantes de la fecundidad para los municipios metropolitanos han sufrido una variación importante. En el año 2001 únicamente el nivel de renta familiar disponible del municipio aparece como variable explicativa significativa (con un coeficiente Beta estandarizado de 0,75). En otras palabras, existía una correlación positiva entre riqueza del municipio e intensidad de la fecundi- 
dad, que era mayor cuando más rico era el municipio. Esta relación, además, es particularmente fuerte en las ciudades (coeficiente de 0,70), mientras que la significatividad del modelo definida a partir de la $\mathrm{R}^{2}$ es de 0,73 .

\section{Cuadro No 2}

Análisis de regresión, resultados para los años 2001 y 2009

\begin{tabular}{|c|c|c|c|c|c|c|}
\hline & & 2001 & & & 2009 & \\
\hline Variables & $\begin{array}{c}\text { Modelo } \\
\text { global }^{*}\end{array}$ & $\begin{array}{c}\text { Modelo } \\
\text { grandes } \\
\text { ciudades }^{\star *} \\
\end{array}$ & $\begin{array}{c}\text { Modelo } \\
\text { significativo ** }\end{array}$ & $\begin{array}{c}\text { Modelo } \\
\text { global*** }\end{array}$ & $\begin{array}{c}\text { Modelo } \\
\text { grandes } \\
\text { ciudades }^{\star *}\end{array}$ & $\begin{array}{c}\text { Modelo } \\
\text { significativo*** }\end{array}$ \\
\hline $\begin{array}{l}\text { Movilidad } \\
\text { residencial } \\
\text { (autóctonos) }\end{array}$ & 0,079 & 0,668 & - & $0,456^{* *}$ & 0,379 & $0,477^{* *}$ \\
\hline $\begin{array}{l}\text { Movilidad } \\
\text { residencial } \\
\text { (extranjeros) }\end{array}$ & 0,197 & 0,317 & - & 0,060 & 0,044 & \\
\hline $\begin{array}{l}\text { Renta familiar } \\
\text { disponible }\end{array}$ & $0,568^{* *}$ & $0,695^{* *}$ & $0,746^{* * *}$ & $-0,351^{*}$ & $-0,337$ & $-0,397^{* *}$ \\
\hline \% europeos & 0,164 & $-0,444$ & & $-0,263^{*}$ & $-0,225$ & $-0,289^{* *}$ \\
\hline$\%$ africanos & 0,133 & 0,037 & & $0,457^{* *}$ & $0,446^{*}$ & $0,441^{* * *}$ \\
\hline$\%$ americanos & $-0,412$ & $-0,390$ & $-0,416^{\star *}$ & $-0,236^{*}$ & $-0,197$ & $-0,237^{*}$ \\
\hline \% asiáticos & $-0,073$ & 0,103 & 1 & $0,317^{*}$ & $0,354^{*}$ & $0,315^{* *}$ \\
\hline $\mathrm{N}$ & 24 & 17 & 24 & 25 & 18 & 25 \\
\hline $\mathrm{R}^{2}$ corregido & 0,343 & 0,730 & 0,382 & 0,804 & 0,749 & 0,814 \\
\hline Significatividad & 0,046 & 0,004 & 0,003 & 0,000 & 0,002 & 0,000 \\
\hline
\end{tabular}

Los niveles de significatividad de cada coeficiente se indican mediante * $p 0.1 /{ }^{* *} p 0.05 /{ }^{* * *} p 0.001$ Nota: Movilidad residencial: Tasas municipales de migración interna neta (calculadas para la población autóctona y la extranjera); Renta familiar disponible: media municipal de la renta de las familias, por habitante en miles de euros; Porcentajes de Europeos, Africanos, Americanos y Asiáticos sobre el total de población de un municipio determinado.

Esta relación entre renta familiar y fecundidad puede, en cierta medida, verse también en el modelo "global" incluso incluyendo municipios por debajo de dicho umbral (coeficiente de renta disponible = $0,57)$. Sin embargo, los modelos que incluyen toda la región metropolitana la $\mathrm{R}^{2}$ no es suficientemente sólida para realizar afirmaciones al respecto.

Fuente: Elaboración propia.

A finales de la década, en 2009, los determinantes de la fecundidad han variado. Por un lado, los municipios de mayor renta disponible ya no son aquellos donde se observan los valores de fecundidad más elevados. Si en 2001 estos municipios tenían altas fecundidades (coeficiente Beta estandarizado de 0,75), en 2009 esta misma variable se correlaciona pero de forma negativa (coeficiente Beta de -0,40). En este mismo año, la migración neta de población con nacionalidad española tiene un efecto positivo importante en los niveles de fecundidad (un coeficiente significativo y del 0,46 en el modelo "global"). Esto podría explicarse por los flujos migratorios internos hacia los municipios de menos de 45 mil habitantes, ya que el modelo para los municipios mayores muestra cómo moverse hacia ellos no es significativo. La formación de hogares relacionada 
con la movilidad residencial se estaría produciéndose hacia los municipios más pequeños, menos densos y suburbanos.

Por el otro lado, los modelos de regresión para 2009 muestran como la fecundidad metropolitana no puede seguir siendo explicada sin tener en cuenta el crecimiento de la población extranjera, como era de esperar, pero también su composición por nacionalidad. Sin embargo, la relación existente no siempre funciona en el mismo sentido. Mientras la presencia de europeos y americanos se correlaciona de forma negativa con el ISF (con valores de -0,29 y -0,24 respectivamente), la presencia de asiáticos y particularmente de africanos mantienen una relación positiva $(0,31$ y 0,44$)$. De hecho, el coeficiente estandarizado de la presencia de africanos es similar al de la migración interna de españoles $(0,48)$, el valor más elevado. La mayor diferencia es la significatividad que adquieren los africanos en el modelo de las ciudades.

Resumiendo, la fecundidad en la RMB pasa de un modelo donde en las grandes ciudades ésta se correlaciona directamente de la renta disponible del hogar, a otro modelo donde el asentamiento de la población extranjera, y en particular de la población africana, es el principal elemento explicativo. La llegada de parejas autóctonas vía migración interna también influencia los niveles de fecundidad, pero únicamente en los municipios medianos y pequeños de la región metropolitana.

\section{Conclusiones y discusión}

La Región Metropolitana de Barcelona es un claro ejemplo de territorio caracterizado por una creciente complejidad, diversidad basada en el continuado proceso de dispersión de la población $y$, al mismo tiempo, de la actividad económica. Esta dinámica, que por un lado construye una región policéntrica e interconectada, por el otro ayuda a fragmentar el territorio metropolitano, acrecentando las diferencias internas. Tanto la población que se asienta en uno u otro municipio, como la población que no se mueve, o la población que se desplaza, elijen el municipio de residencia condicionados por sus características socioeconómicas, debido a que el territorio no es, en términos sociales, indiferente. Como resultado, además del crecimiento de unas u otras áreas, se construyen espacios cada vez más heterogéneos, donde las diferencias socioeconómicas pero también sociodemográficas se manifiestan. Este es el caso aquí planteado, el del crecimiento de las desigualdades demográficas internas metropolitanas. Las diferencias entre los municipios con fecundidades elevadas y con fecundidades bajas son cada vez más importante, y se producen en un contexto donde estas diferencias, a nivel estatal, se están reduciendo. Existe mayor diferencia entre dos municipios vecinos que entre dos provincias alejadas, reflejo de la fragmentación metropolitana actual, por un lado, y del acercamiento de comportamientos entre regiones, por el otro.

Entre los municipios, y en función de su fecundidad, se pueden fácilmente distinguir tres grandes tipologías, definidas por su centralidad, por la presencia de ciertos colectivos extranjeros, y por la migración interna de parejas jóvenes. En primer lugar, y con independencia de la dinámica general, destaca el caso de la ciudad de Barcelona, con una fecundidad muy baja y en niveles prácticamente similares durante los últimos 25 años. Desde finales de los ochenta a finales de los noventa Barcelona, a pesar de la baja fecundidad, no experimenta el descenso que sí que observan sus ciudades adyacentes, manteniéndose por encima, incluso en la media del conjunto catalán en los años 1996 y 1997. Tampoco, en los años de crecimiento de la fecundidad, Barcelona 
participa de esta dinámica, situándose poco a poco como la ciudad de menor fecundidad de la metrópolis. Los efectos de la Segunda Transición Demográfica, que se dan en primer lugar en la ciudad central, pueden explicar algunas de las diferencias en la intensidad encontradas, y también en su tardío calendario. Mayores proporciones de parejas sin hijos, de parejas monoparentales, o de formas familiares más complejas pueden ejemplificar el mayor peso de este proceso en la capital. El filtro demográfico de la ciudad, expulsando parejas jóvenes y atrayendo jóvenes solos y de mayor nivel educativo, puede también explicar la dinámica de la ciudad central. En cambio, la presencia de extranjeros, también mayor que en la mayoría de la periferia, no se traduce en un aumento de la fecundidad. Las parejas extranjeras, en este caso, actúan como las autóctonas y prefieren los municipios suburbanos para reproducirse. Lo que nos falta por explicar es el porqué del nivel de fecundidad observado. Es decir, porqué la fecundidad se mantiene estable alrededor de los 1,15-1,25 hijos por mujer, y no significativamente por debajo o por encima.

Los municipios cercanos a la ciudad central, predominantemente de clase trabajadora y caracterizados por bajos niveles de renta, muestran una pauta divergente. Son municipios bien conectados con transporte público y con vivienda más asequible, que conocen primero una fuerte emigración y después una entrada importante de extranjeros. Estos son los más sensibles a la coyuntura económica, capaces de mostrar en pocos años las fecundidades más elevadas y después las más bajas. En la actualidad, y a diferencia de la ciudad central, la presencia elevada de extranjeros hace aumentar sus niveles de fecundidad, en mayor medida cuando los extranjeros son africanos o asiáticos, en menor intensidad si son europeos o latinoamericanos.

Finalmente, el tercer gran grupo de municipios, situados mayoritariamente en la periferia, son los que experimentan fecundidades más elevadas, debido a que reciben elevados números de parejas autóctonas vía movilidad residencial, al mismo tiempo que los extranjeros allí residentes son, en su mayoría, africanos. En este último grupo se podría distinguir entre ciudades de cierto tamaño (por encima de 45 mil habitantes) y municipios menores. El asentamiento en el primer caso se produce en viviendas plurifamiliares, y la población extranjera tiene un peso significativo, mientras en el segundo son las viviendas unifamiliares las que reciben a la nueva población.

Estos resultados, deducidos del análisis descriptivo, aparentemente verifican nuestra hipótesis de trabajo, es decir, que existe un incremento de las diferencias en la fecundidad debido a la migración interna selectiva. Una segunda hipótesis de trabajo, apoyada en la idea de que los niveles de población extranjera influencian los niveles de fecundidad, podría ser validada en parte, a excepción de la ciudad central. En muchos municipios ambos factores se dan al mismo tiempo, con lo que las diferencias se incrementan.

El análisis de regresión confirma los resultados descriptivos. Para el último año, 2009, situado al final de una década de alta intensidad migratoria y de crecimiento económico hasta 2008, las variables explicativas de la fecundidad son las migraciones internas de los nativos hacia las periferias y las pautas de asentamiento de los inmigrantes extranjeros, aunque en este caso no de todos ellos, y si de los de ascendencia africana y asiática. Esto explica por qué municipios como Santa Coloma de Gramenet aumentan su fecundidad y otros, con porcentajes de extranjeros similares, como I'Hospitalet de Llobregat, tienen un ISF menor. El tercer factor analizado, la renta familiar disponible por municipio, se encontraba positivamente relacionada con la fecundidad a inicios de 2001 (también en años anteriores), pero en cambio su papel no es determinante en el año 2009. 
En este caso, cabe considerar la alta heterogeneidad en los restos comarcales, pero también en el interior de las grandes ciudades.

Los resultados aquí obtenidos son similares a los de otros países europeos, confirmando tanto la hipótesis contextual como la de la selección de migrantes, ambas serian válidas para explicar las diferencias en la fecundidad. La tercera hipótesis, la composicional, no podemos a partir de nuestro análisis aceptarla ni refutarla, aunque el bajo nivel de fecundidad de la ciudad central, y el inmovilismo de sus valores, pudieran hacernos pensar en su validez. Finalmente, recalcar como el crecimiento de la diversificación residencial y la fragmentación urbana tiene un efecto en el comportamiento demográfico bastante visible, que puede actuar incrementando las diferencias de partida. El análisis en profundidad de estas diferencias y de sus causas pueden ayudar en la planificación territorial, afinando en las proyecciones de población para áreas pequeñas, pero también considerando que no todos los territorios tienen exactamente las mismas necesidades.

\section{Referencias bibliográficas}

AGUILAR, G.A. (coordinador). Procesos metropolitanos y grandes ciudades. Dinámicas recientes en Máxico y otros países. México: UNAM, Instituto de Geografía, 2004.

AGUILAR, A.G. y MATEOS, P. Diferenciación sociodemográfica del espacio urbano de la Ciudad de México. EURE, 2011, Vol. 37, No 110, p. 5-30.

ALBERICH, J. L'ús de l'espai. Papers de la RMB, 2010, Vol. 51, p. 28-43.

ALBRECHT, D.R. y ALBRECHT, C.M. Metro/nonmetro residence, nonmarital conception, and conception outcomes. Rural Sociology, 2004, Vol. 69, No 3, p. 430-452.

BAYONA, J. \& GIL-ALONSO, F. Suburbanisation and international immigration: The case of the Barcelona Metropolitan Region (1998-2009). Tijdschrift voor Economische en Sociale Geografie, 2012, Vol. 103, No 3, p. 312-329.

BAYONA, J. y PUJADAS, I. Movilidad residencial y redistribución de la población metropolitana: los casos de Madrid y Barcelona. EURE, 2014, Vol. 40, No 119, p. 261-287.

BERGER, M. Les périurbains de Paris. De la ville dense à la ville éclatée? París: CNRS, 2004.

BORSDORF, A. Cómo modelar el desarrollo y la dinámica de la ciudad latinoamericana. Revista EURE, 2003, Vol. XXIX, No 86, p. 37-49.

BOYLE, P. Population geography: does geography matter in fertility research? Progress in Human Geography, 2003, Vol. 27, No 5, p. 615-626.

BRUNETTA, G. \& ROTONDI, G. Urban and rural fertility in Italy: regional and temporal changes. In: BÄHR, J. y GANS, P. (editors). The Geographical Approach to Fertility. Kiel: Geographischer Institut der Universität Kiel, 1991, p. 203-217. 
CHAMPION, A.G. Urbanization, Suburbanization, Counterurbanization and Reurbanization. In: PADDISON, R. (editor). Handbook of Urban Studies. London: SAGE, 2001, p. 143-161.

CHAMPION, A.G. Population change and migration in the British urban system. In: GEYER, H.S. (editor). International handbook of urban system: studies of urbanization and migration in advanced and developing countries. Cheltenham: Edward Elgar Publishing, 2002, p. 87-120.

CHESIRE, P. Resurgent cities, urban myths and policy hubris: what we need to know. Urban studies, 2006, Vol. 43, No 8, p. 1231-1246.

COALE, A.J. \& HOOVER, E.M. Population Trends and Economic Development in Low-Income Countries. Princeton: Princeton University Press, 1958.

CASTRO, T. y ROSERO-BIXBY, L. Maternidades y fronteras: La fecundidad de las mujeres inmigrantes en España. Revista Internacional de Sociología, 2011, p. 105-137.

COSTA, M. \& PORCEL, S. L'estructura socioespacial de la regió metropolitana de Barcelona des d'una aproximació multimètode. Una proposta metodològica per a l'estudi de la diferenciació socioresidencial. Documents d'Anàlisi Geogràfica, 2013, Vol. 59, No 2, p. 315-345

COURGEAU, D. Family Formation and Urbanisation. Population: an English Selection, 1989, Vol. 44, No 1, p. 123-146.

DEMATTEIS, G. Suburbanización y periurbanización. Ciudades anglosajonas y ciudades latinas. En: MONCLÚS, F.J. (editor). La ciudad dispersa. Barcelona: Centre de Cultura Contemporània, 1998, p. 17-34.

DEVOLDER, D. y BUENO, X. Interacciones entre fecundidad y migración. Un estudio de las personas nacidas en el extranjero y residentes en Cataluña en 2007. Documents d'Anàlisi Geogràfica, 2011, Vol. 57, No 3, p. 441-467.

DEVOLDER, D. y CABRÉ, A. Factores de la evolución de la fecundidad en España en los últimos 30 años. Panorama Social, 2009, Vol. 10, p. 23-39.

DOMINGO, A.; BAYONA, J. y LÓPEZ-GAY, A. Impacto demoespacial de la internacionalización de los flujos migratorios en la ciudad de Barcelona. Migraciones, 2004, Vol. 16, p. 157-198.

ESTEBAN, J. La Regió Metropolitana de Barcelona. Revista Papers, 2003, Vol. 39, p. 31-41.

FAGNANI, J. Fertility in France: the influence of urbanization. In: BÄHR, J. y GANS, P. (editors). The Geographical Approach to Fertility. Kiel: Geographischer Institut der Universität Kiel, 1991, p. 165173.

FERIA, J.M. Ciudad y Territorio. Nuevas dinámicas espaciales. En: PUJADAS, I. et al. (editores). Población y Espacios Urbanos. Barcelona: Departament de Geografía Humana de la UB y Grupo de población de la AGE, 2011, p. 13-52. 
FIELDING, A.J. Population redistribution in Western Europe: trends since 1950 and the debat about Counterurbanization. En: CONDONG, P. \& BATEY, P. (editors). Advances in regional demography: information, forecats, models. London: Belhaven Press, 1989, p. 167-179.

FIORI, F.; GRAHAM, E. \& FENG, Z. Geographical variations in fertility and transition to second and third birth in Britain. Advances in Life Course Research, 2014, No 21, p. 149-167

GALEANO, J.; SABATER, A. \& DOMINGO, A. Formació i evolució dels enclavaments ètnics a Catalunya abans i durant la crisi econòmica. Documents d'Anàlisi Geogràfica, 2014, Vol. 60, № 2, p. 261-288.

GIL-ALONSO, F.; BAYONA, J. \& PUJADAS, I. From boom to crash: Spanish urban areas in a decade of changes (2001-2011). European Urban and Regional Studies, 2016, Vol. 23, No 2, p. 198-216.

GIL-ALONSO, F.; BAYONA, J.; PUJADAS, I. y LÓPEZ, C. Diferenciación espacial de la fecundidad en España: la creciente diversidad en el interior de las provincias. En: CAIRO, H. y FINKEL, L. (coordinaroes). Actas del XI Congreso Español de Sociología. Crisis y cambio: propuestas desde la Sociología, Vol. III, Madrid: FES, 2014, p. 73-85.

HANK, K. Regional fertility differences in Western Germany: an overview of the literature and recent descriptive findings. International Journal of Population Geography, 2001, Vol. 7, No 4, p. 243-257.

HARVEY, D. Social justice and the city. Athens: University of Georgia Press, 2010.

INDOVINA, F.; BONETA, X. y HERRERO, M. (coordinadores). La ciudad de baja densidad. Barcelona: Diputació de Barcelona, 2007.

$\mathrm{KABISCH}, \mathrm{N}$. \& HAASE, D. Diversifying European Agglomerations: Evidence of Urban Population Trends for the 21st Century. Population, Space and Place, 2011, No 17, p. 236-253.

KULU, H. Why do Fertility Levels Vary between Urban and Rural Areas? Regional Studies, 2013, Vol. 47, № 6, p. 895-912.

KULU, H. \& BOYLE, P. High Fertility in City Suburbs: Compositional or Contextual Effects?. European Journal of Population, 2009, Vol. 25, p. 157-174.

KULU, H. \& VIKAT, A. Fertility differences by housing type: The effect of housing conditions or of selective moves? Demographic Research, 2007, Vol. 17, No 26, p. 775-802.

KULU, H.; BOYLE, P. \& ANDERSSON, G. High suburban fertility: Evidence from four Northern European countries. Demographic Research, 2009, Vol. 21, p. 915-944.

LEAL, J. Segregación social y mercado de vivienda en las grandes ciudades. Revista Española de Sociología, 2003, № 2, p. 57-75. 
LÓPEZ, C.; PUJADAS, I. \& BAYONA, J. Households within the residential mobility process: The case of Barcelona metropolitan region. Archivio di Studi Urbani e Regionali, 2013, Vol. 108, p. 57-84.

MILEWSKI, N. First child of immigrant workers and their descendants in West Germany: Interrelation of events, disruption, or adaptation? Demographic Research, 2007, Vol. 17, p. 859-896.

MÓDENES, J.A. Flujos espaciales e itinerarios biográficos: la movilidad residencial en el área de Barcelona. Barcelona: Mimeo / Tesis Doctoral, Departamento de Geografia, Universitat Autònoma de Barcelona, 1998.

MÓDENES, J.A.; BAYONA, J. \& LÓPEZ-COLÁS, J. Immigration and Residential Change in Spain. Population, Space and Place, 2013, Vol. 19, No 3, p. 294-310.

MONCLÚS, F.J. (editor). La ciudad dispersa. Suburbanización y nuevas periferias. Barcelona: Centre de Cultura Contemporània, 1998.

MULDER, C.H. Population and housing: a two-sided relationship. Demographic Research, 2006, Vol. 15, p. 401-412.

NOTESTEIN, F.W. Economic problems of population change. London: Oxford University Press, 1953.

POZO, E. y RODRÍGUEZ-MOYA, J.M. Diferencias socioespaciales en la fecundidad madrileña en el cambio de siglo. Estudios Geográficos, 2003, Vol. LXIV, No 252, p. 455-486.

PUJADAS, I. Movilidad residencial y expansión urbana en la región metropolitana de Barcelona, 1982-2005, Scripta Nova. Revista Electrónica de Geografía y Ciencias Sociales, 2009, Vol. XIII, No 290. Disponible en Internet: http://www.ub.edu/geocrit/sn/sn-290.htm

PUJADAS, I.; BAYONA, J.; GIL-ALONSO, F. y LÓPEZ, C. Pautas territoriales de la fecundidad en la Región Metropolitana de Barcelona (1986-2010). Estudios Geográficos, 2013, vol. LXXIV, № 275, p. 585-609.

PUJADAS, I.; LÓPEZ, C. \& BAYONA, J. Residential mobility in the Barcelona Metropolitan Region during the present economic crisis, PortugueseJournal of Social Sciences, 2016, Vol. 15, N01, p. 91-110.

PUJADAS, I.; PRATS, P. y COLL, M. Elección residencial y nuevas formas urbanas: El caso de la Región Metropolitana de Barcelona. En: Los procesos urbanos postfordistas. VIII Coloquio de Geografía Urbana. Palma: Servei de Publicacions de la Universitat de les Illes Balears, 2007, p. 215-234.

PUJADAS, I. La fecunditat. En: Enquesta de la Regió Metropolitana de Barcelona 1990. Condicions de vida i hàbits de la población. Barcelona: IEMB, 1992, p. 51-69.

RÉRAT, P. The New Demographic Growth of Cities: The case of Reurbanisation in Switzerland. Urban Studies 2012, Vol. 49, No 5, p. 1107-1125. 
ROCA, J.; ARELLANO, B. y MOIX, M. Estructura urbana, policentrismo y sprawl: los ejemplos de Madrid y Barcelona. Ciudad y territorio, 2011, Vol. XLIII, No 168, p. 299-321.

RUBIALES, M.; BAYONA, J. y PUJADAS, I. Patrones espaciales de la segregación residencial en la Región Metropolitana de Barcelona: Pautas de segregación de los grupos altos. Scripta Nova, Revista Electrónica de Geografía y Ciencias Sociales, 2012, Vol. XVI, No 423. Disponible en Internet: http://www.ub.edu/geocrit/sn/sn-423.htm

SABATINI, F. y BRAIN, I. La segregación, los guetos y la integración social urbana: mitos y claves. EURE, 2008, Vol. XXXIV, No 103, p. 5-26 
